\title{
麦克纳姆轮驱动的移动机器人自适应 滑模控制器设计
}

\author{
王明明 ${ }^{1,2}$ ，朱莹莹 ${ }^{3}$, 张磊 ${ }^{1}$ ，王璐 ${ }^{1}$ ，卫宣伯 ${ }^{1}$ ，方静 ${ }^{1}$
}

$\left.\begin{array}{l}\text { 1.西北工业大学 航天学院, 陕西 西安 710072; 2. 西北工业大学 深圳研究院, 广州 深圳 } 518057 \text {; } \\ \text { 3.武警特指挥学院, 北京 } 102202\end{array}\right)$

\begin{abstract}
摘 要: 针对麦克纳姆全向轮驱动的移动机器人轨迹跟踪控制问题,设计了一种自适应滑模控制器。 将自适应鲁棒控制应用于麦克纳姆轮驱动的移动机器人轨迹跟踪以获得良好的动态跟踪性能以及鲁 棒性能。首先对基于麦克纳姆轮的移动机器人进行了运动学建模, 在此基础上进行了自适应鲁棒控 制器设计。提出了一种比例一积分一微分 (PID) 形式的滑模面, 满足了系统的鲁棒性要求; 设计了一种 能够快速收敛的趋近律,减少了整定参数所消耗的时间并能有效抵抗外部扰动。最后,存在脉冲扰动 以及正弦信号扰动条件下对控制器进行了仿真验证, 证明了所提控制器的优越性, 通过轨迹跟踪控制 的样机试验,证明了该方法的实用性和可靠性。
\end{abstract}

\section{关 键 词: 轨迹跟踪; 控制器; 鲁棒控制器; 自适应; 滑模控制; 麦克纳姆轮 \\ 中图分类号:TP242.6 \\ 文献标志码:A \\ 文章编号: 1000-2758(2018)04-0627-09}

移动机器人在复杂地形探测、战场侦查、地质勘 探、仓储物流等方面具有重要的应用前景, 其控制器 的设计水平是保证任务成功的关键 ${ }^{[1]}$ 。机器人控 制器的设计开始向着应用于复杂环境, 且能抵抗外 部干扰的方向发展。由于能全向移动且转弯半径为 零, 基于全向轮的移动机器人受到了广泛关注, 并且 被应用于多种场景, 如仓库搬运机器人、全向轮椅 等。全向移动机器人中, 麦克纳姆轮驱动的全向移 动机器人受到越来越多的关注 ${ }^{[2-3]}$ 。

由于麦克纳姆轮驱动的全向移动机器人存在诸 多优势, 使其在工业界和学术界得到越来越多的关 注, 诸多学者对其运动学建模和动力学建模展开了 研究 ${ }^{[3]}$ 。Muir 等 ${ }^{[4]}$ 对此类机器人进行了运动学建 模, 从运动学方式上, 给出了基于麦克纳姆轮的全向 机器人运动学公式。Tlale 等 ${ }^{[5-6]}$ 为此类机器人建立 了动力学模型并从动力学角度对基于麦克纳姆轮的 全向机器人展开分析。尽管此类机器人的运动学和 动力学模型发展较为成熟, 针对应用于实际环境的

\section{收稿日期: 2017-05-20}

移动机器人轨迹跟踪控制研究仍然方兴未艾。

由于 4 个麦克纳姆轮分别由 4 个直流电机驱 动, 针对此类机器人如何设计多驱动电机的协调控 制器成为至关重要的问题。实际工程应用中, 电机 选型多选用带有速度伺服系统的电机, 由于电机的 速度伺服系统多采用 PID 控制, 因此伺服控制系统 会对整个系统带来更多的不确定性; 此外机器人所 运行的环境也具有诸多不确定性, 因此控制器的鲁 棒性和自适应性就显得至关重要。Shimada 等 ${ }^{[7]}$ 提 出了一种基于位置误差的控制方法, 并研制了实验 装置, 对所提出的方法进行了实验验证。Wang 等 ${ }^{[8]}$ 在不考虑扰动的情况下, 提出了一种用于轨迹 规划的滑动模态控制器, Viet 等 ${ }^{[9]}$ 考虑了有扰动的 情况, 在外加扰动的情况下针对全向移动机器人设 计了滑模控制器, 其研究结果表明滑模控制器能有 效抑制外部干扰。Fierro 等 ${ }^{[10]}$ 针对全向移动机器人 的轨迹规划设计了一种神经网络控制器。另外, $\mathrm{Xu}$ 等 ${ }^{[11]}$ 针对全向移动机器人设计了一种基于神经网 
络的鲁棒滑模控制器。基于神经网络的控制器具有 控制精度高等优点, 但需要大量学习, 因此具体实现 上存在不便。以上控制器存在参数难以整定的问 题, 如何设计一种高效的自适应控制器是解决此问 题的关键。Wang 等 ${ }^{[12]}$ 针对移动机器人的轨迹规划 设计了一种自适应控制器, 并证明了其稳定性。 Sira-Ramirez 等 ${ }^{[13]}$ 设计了一种能够快速收敛的自适 应控制器, 以应对直流电机的不确定性。

综上所述, 本文的研究对象具有确定的运动学 模型,适合采用基于模型的控制方法。常见的基于 模型的控制方法有: LQR 控制方法 ${ }^{[14]}$ 以及结合 LQR 控制方法优点与卡尔曼滤波器的控制方法 LQG 控制 ${ }^{[15]}$, 然而 LQR 控制虽然具有良好的鲁棒 性,但其计算复杂且对模型精确度要求过高。而 LQG 控制鲁棒性较差, 并且该类方法多用于线性系 统控制器设计。通过对移动机器人的运动学建模可 知,基于麦克纳姆轮的全向移动机器人是一类多输 人多输出 (MIMO) 的非线性系统, 而滑动模态控 制 ${ }^{[16]}$ 是一种针对不确定条件且具有鲁棒性的非线 性控制器。因此本文提出基于滑模控制的自适应鲁 棒轨迹跟踪控制器。滑模控制器的常见问题为控制 器输出的抖振问题, 马广富等 ${ }^{[17]}$ 提出了一种多幂次 滑模趋近律, 能够显著减小抖振, 并且通过仿真进行 了验证,但其不具有自适应性。为使系统具有更高 的鲁棒性,本文设计了一种比例-微分-积分形式的 滑模面来提高系统的鲁棒性, 并且提出了一种多幂 次趋近律来提高系统的动态性能, 最后为避免参数 整定的麻烦操作, 本文设计了自适应律以提高系统 的自适应性,借此可以解决上述问题。

\section{1 运动学模型}

麦克纳姆轮是一种在轮表面存在一些滚子 (如 图 1 所示)。

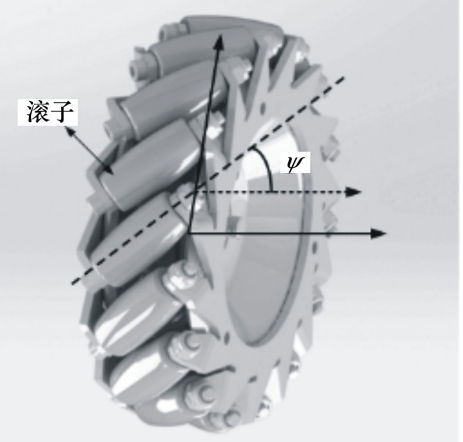

这些滚子的转轴方向与整个轮的转轴方向呈 $45^{\circ}$ 夹角, 每个麦克纳姆轮由一个独立的直流电机驱 动, 通过调节 4 只电机的不同转速, 即可实现全向的 移动, 比如横向和纵向移动, 倾斜移动和零转弯角度 转动 (如图 2 所示)。

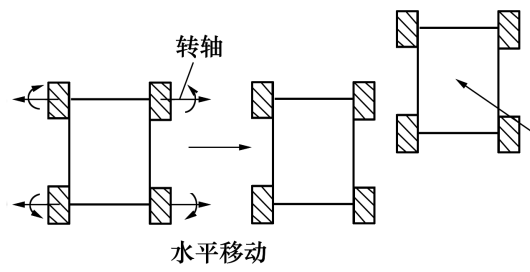

倾斜移动

图 2 带麦克纳姆轮的移动机器人运动演示

对麦克纳姆轮驱动的全向移动机器人进行运动 学建模, 首先要建立相关的坐标系 (如图 3 所示), $O_{i} x_{i} y_{i}$ 为惯性坐标系 (inertial coordinate system), $O_{b} x_{b} y_{b}$ 为连体坐标系 (body-fixed coordinate system), 其原点 $O_{b}$ 为车体的几何中心, 其 $y$ 轴始终 指向车体纵轴, $x$ 轴与 $y$ 轴垂直并组成右手坐标系, $O_{w i} x_{w i} y_{w i}$ 为第 $i$ 只轮子的轮系坐标系, 其原点位于麦 克纳姆轮的几何中心, $x$ 轴与驱动电机输出轴平行, $y$ 轴与 $x$ 轴垂直并组成右手坐标系。规定车体右前 方的轮子为 1 号轮,顺序按逆时针排序。

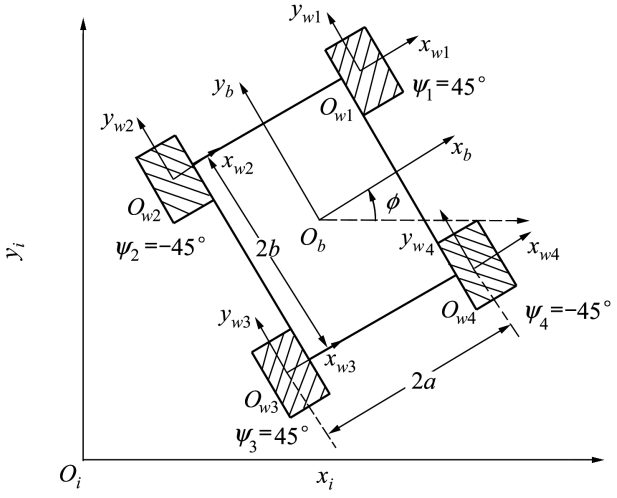

图 3 移动机器人坐标系

如图 1 所示, 定义麦克纳姆轮滚子的转轴与 $O_{w i} x_{w i} y_{w i}$ 的 $x$ 轴所呈夹角为 $\psi$, 定义第 $i$ 只轮 $O_{w i} x_{w i} y_{w i}$ 位置矢量 $\boldsymbol{p}_{w i}(i=1,2,3,4)=\left[x_{w i}, y_{w i}, z_{w i}\right]^{\mathrm{T}}, \omega_{i x}$ 为第 $i$ 只轮的角速度, $\omega_{i v}$ 为第 $i$ 只轮上的滚子角速度, $\omega_{i z}$ 为麦克纳姆轮绕过 $O_{w i}$ 且垂直于 $O_{w i} x_{w i} y_{w i}$ 平面转轴 的角速度。 $R_{i}(i=1,2,3,4)$ 为第 $i$ 只轮子的半径, $r_{i}$ 为滚子半径, 轮系坐标系中轮子的速度矢量可以表 示为: 


$$
\boldsymbol{p}_{w i}=\left[\begin{array}{c}
\dot{x}_{w i} \\
\dot{y}_{w i} \\
\dot{\varphi}_{w i}
\end{array}\right]=\left[\begin{array}{ccc}
0 & r \sin \left(\psi_{i}\right) & 0 \\
R_{i} & -r \cos \left(\psi_{i}\right) & 0 \\
0 & 0 & 1
\end{array}\right]\left[\begin{array}{c}
\omega_{i x} \\
\omega_{i y} \\
\omega_{i z}
\end{array}\right]
$$

根据旋转矩阵, 可以获得如 (2) 式所示的速度 计算公式:

$$
\begin{gathered}
\dot{\boldsymbol{p}}_{b}=\boldsymbol{J}_{i} \dot{\boldsymbol{q}}_{i} \\
\boldsymbol{J}_{i}=\left[\begin{array}{ccc}
0 & r_{i} \sin \left(\psi_{i}\right) & d_{w i y}^{b} \\
R_{i} & -r_{i} \cos \left(\psi_{i}\right) & d_{w i x}^{b} \\
0 & 0 & 1
\end{array}\right]
\end{gathered}
$$

式中, $\boldsymbol{p}_{b}$ 为移动机器人在 $O_{b} x_{b} y_{b}$ 中的速度矢量, $\boldsymbol{J}_{i}$ 是移动机器人关于第 $i$ 只轮的雅可比矩阵, 其中 $d_{w i y}^{b}$ 和 $d_{w i x}^{b}$ 是坐标系 $O_{b} x_{b} y_{b}$ 和坐标系 $O_{w i} x_{w i} y_{w i}$ 之间的平 移距离, $\dot{\boldsymbol{q}}_{i}=\left[\begin{array}{lll}w_{i x} & w_{i y} & w_{i z}\end{array}\right]^{\mathrm{T}}$ 。

对雅可比矩阵分析可以得出如下结论:

1) 当且仅当 $\psi_{i}=0$ 时, $\left|\boldsymbol{J}_{i}\right|=0$ 。所以对于麦 克纳姆轮来说, 此时雅可比矩阵是奇异的。

2 ) 值得注意的是, $\operatorname{rank}\left(\boldsymbol{J}_{i}\right)=3$, 所以每个轮子 具有 3 个自由度。

在本文模型中, 每个麦克纳姆轮规格相同, 因此 $R_{1}=R_{2}=R_{3}=R_{4}=R, r_{1}=r_{2}=r_{3}=r_{4}=r, d_{w 1 x}^{b}=a, d_{w 1 y}^{b}$ $=b, d_{w 2 x}^{b}=-a, d_{w 2 x}^{b}=b, d_{w 3 x}^{b}=-a, d_{w 3 y}^{b}=-b, d_{w 4 x}^{b}=a$, $d_{w 4 y}^{b}=-b$, 针对每个轮子可以列写其雅可比矩阵 如下:

$$
\begin{gathered}
\boldsymbol{J}_{1}=\left[\begin{array}{ccc}
0 & r / \sqrt{2} & b \\
R & -r / \sqrt{2} & a \\
0 & 0 & 1
\end{array}\right] \quad \boldsymbol{J}_{2}=\left[\begin{array}{ccc}
0 & -r / \sqrt{2} & b \\
R & -r / \sqrt{2} & -a \\
0 & 0 & 1
\end{array}\right] \\
\boldsymbol{J}_{3}=\left[\begin{array}{ccc}
0 & r / \sqrt{2} & -b \\
R & -r / \sqrt{2} & -a \\
0 & 0 & 1
\end{array}\right] \quad \boldsymbol{J}_{4}=\left[\begin{array}{ccc}
0 & -r / \sqrt{2} & -b \\
R & -r / \sqrt{2} & a \\
0 & 0 & 1
\end{array}\right]
\end{gathered}
$$

通过 (2) 式和 (3) 式, 移动机器人的微分运动学 公式可以描述如下:

$$
\left[\begin{array}{l}
\dot{x}_{r} \\
\dot{y}_{r} \\
\dot{\varphi}_{r}
\end{array}\right]=\frac{R}{4}\left[\begin{array}{cccc}
-1 & 1 & -1 & 1 \\
1 & 1 & 1 & 1 \\
\frac{1}{a+b} & \frac{-1}{a+b} & \frac{-1}{a+b} & \frac{1}{a+b}
\end{array}\right] \times\left[\begin{array}{l}
\omega_{1} \\
\omega_{2} \\
\omega_{3} \\
\omega_{4}
\end{array}\right]
$$

式中, $\omega_{i}(i=1,2,3,4)$ 是第 $i$ 只轮的转速, 单位为 $\mathrm{rad} / \mathrm{s}$ 。通过运动学公式不难发现, 基于麦克纳姆轮 的移动机器人可以实现真正的全向移动。
闭环控制的反馈为静止坐标系下的位姿信息。 进一步地, 根据旋转矩阵, 可得到连体坐标系下的速 度 $O_{b} x_{b} y_{b}$ 和惯性坐标系下的速度 $O_{i} x_{i} y_{i}$ 的转换关 系为:

$$
\begin{gathered}
\dot{\boldsymbol{P}}_{i}=\left[\begin{array}{lll}
\dot{x}_{q} & \dot{y}_{q} & \dot{\boldsymbol{\varphi}}_{q}
\end{array}\right]^{\mathrm{T}}=\boldsymbol{\Gamma}(\varphi) \dot{\boldsymbol{P}}_{r}= \\
{\left[\begin{array}{ccc}
\cos (\varphi) & -\sin (\varphi) & 0 \\
\sin (\varphi) & \cos (\varphi) & 0 \\
0 & 0 & 1
\end{array}\right] \dot{\boldsymbol{P}}_{r}}
\end{gathered}
$$

\section{2 自适应滑模控制器设计}

\section{1 滑模面设计}

滑模控制器设计的第一个任务就是设计滑模 面,在控制系统工作的过程中, 保证系统始终工作在 滑模面 $\boldsymbol{s}(t)=\mathbf{0}$ 上。

首先定义误差向量为 $: \boldsymbol{e}(t)=\left[\begin{array}{lll}e_{1} & e_{2} & e_{3}\end{array}\right]^{\mathrm{T}}$, 轨 迹的期望位姿为 $\boldsymbol{p}_{d}(t)=\left[\begin{array}{lll}x_{d} & y_{d} & \boldsymbol{\varphi}_{d}\end{array}\right]^{\mathrm{T}}$, 实际位姿 为 $\boldsymbol{p}_{i}(t)=\left[\begin{array}{lll}x_{i} & y_{i} & \boldsymbol{\varphi}_{i}\end{array}\right]^{\mathrm{T}}, \boldsymbol{e}(t)=\boldsymbol{p}_{d}(t)-\boldsymbol{p}_{i}(t)$ 。显然 地, 滑模面是关于误差的函数, 目前常用的滑模面是 比例 - 微分形式的滑模面, 此前的研究表明, 滑模 面中积分项的引人会显著地提高系统的鲁棒性, 因 此本文选取一种比例 - 微分 - 积分形式的滑模面 用于提高系统的鲁棒性 ${ }^{[18]}$, 同时可以适应不同的 情况。滑模面设计如下:

$$
\begin{aligned}
\boldsymbol{s}(t)= & \boldsymbol{k}_{p} \boldsymbol{e}(t)+\boldsymbol{k}_{i} \int \boldsymbol{e}(t) \mathrm{d} t+\boldsymbol{k}_{d} \dot{\boldsymbol{e}}(t) \\
& \left\{\begin{array}{l}
\boldsymbol{k}_{p}=\operatorname{diag}\left(k_{p 1}, k_{p 2}, k_{p 3}\right) \\
\boldsymbol{k}_{i}=\operatorname{diag}\left(k_{i 1}, k_{i 2}, k_{i 3}\right) \\
\boldsymbol{k}_{d}=\operatorname{diag}\left(k_{d 1}, k_{d 2}, k_{d 3}\right)
\end{array}\right.
\end{aligned}
$$

\section{2 趋近律设计}

在滑模控制器中, 常用的趋近律有: 等速趋近 律、指数趋近律、幕次趋近律、一般趋近律 ${ }^{[12,18]}$ 。其 中幂次趋近律具有较好的收玫速度, 更进一步地, 为 了保证较高的收玫速度, 并且尽量减小控制器的抖 振问题,本文设计一种多幂次滑模趋近律以达到控 制目标, 并通过仿真验证其在系统动态响应过程中 具有快速的收敛速度。本文选用的多幂次滑模趋近 律如下:

$$
\begin{gathered}
s_{i}(t)=-\varepsilon_{i}\left|s_{i}(t)\right|{ }^{\alpha} \operatorname{sgn}\left(s_{i}(t)\right)-\sigma_{i}\left|s_{i}(t)\right|{ }^{\beta} s_{i}(t) \\
(i=1,2,3)
\end{gathered}
$$

式中 


$$
\operatorname{sgn}\left(s_{i}\right)= \begin{cases}1 & s_{i}>0 \\ -1 & s_{i}<0\end{cases}
$$

符号函数 $\operatorname{sgn}($ ) 的引人会使系统的工作过程出现 振荡。因此, 为了减小符号函数对系统的影响, 本文 对趋近律进行如下改进, 引人饱和函数, 可有效削弱 趋近律在滑模面附近的跳变, 以改善抖振问题:

$$
\dot{s}_{i}(t)=-\varepsilon_{i}\left|s_{i}(t)\right|{ }^{\alpha} \operatorname{sat}\left(s_{i}(t)\right)-\sigma_{i}\left|s_{i}(t)\right|{ }^{\beta} s_{i}(t)
$$

式中, $\delta_{i}$ 为正小量:

$$
\operatorname{sat}\left(s_{i}\right)=\frac{s_{i}}{\left|s_{i}\right|+\delta_{i}}
$$

稳定性证明如下: 首先选取李雅普诺夫函数

$$
\begin{aligned}
& V_{1}(t)=\frac{1}{2} \boldsymbol{s}(t){ }^{\mathrm{T}} \boldsymbol{s}(t)>0 \\
& \dot{V}_{1}(t)=\boldsymbol{s}(t){ }^{\mathrm{T}} \boldsymbol{s}(t)=\sum_{i=1}^{3} s_{i}(t) \dot{\boldsymbol{s}}(t)= \\
& \sum_{i=1}^{3}-\varepsilon_{i}\left|s_{i}(t)\right|{ }^{\alpha} s_{i}(t) \operatorname{sat}\left(s_{i}(t)\right)- \\
& \sigma_{i}\left|s_{i}(t)\right|{ }^{\beta} s_{i}^{2}(t) \leqslant 0
\end{aligned}
$$

当且仅当 $s_{i}(t)=0,(i=1,2,3)$ 时等号成立, 故 该系统是李雅普诺夫稳定的。

\section{3 自适应律设计}

由于环境的不确定性和电机速度伺服系统的不 确定性, 自适应律的参数需要不断整定, 为避免后续 的参数整定, 本文为所设计的滑模控制器设计了专 门的自适应律, 以提高控制器的自适应性。公式 (10) 中的参数 $\varepsilon_{i}$ 会对系统的性能产生比较大的影 响。因此, 为该参数设计自适应律是十分必要的, 规 定 $\hat{\varepsilon}_{i}$ 为参数 $\varepsilon_{i}$ 的估计值, $\stackrel{\varepsilon}{i}_{i}$ 为参数 $\varepsilon_{i}$ 的名义值, $\widetilde{\varepsilon}_{i}=$ $\hat{\varepsilon}_{i}-\stackrel{\leftrightarrow}{\varepsilon}_{i}$ 。这样趋近律可以改写为如下形式:

$$
\begin{gathered}
\dot{s_{i}}(t)=-\hat{\varepsilon}_{i}\left|s_{i}(t)\right|{ }^{\alpha} \operatorname{sgn}\left(s_{i}(t)\right)- \\
\sigma_{i}\left|s_{i}(t)\right|{ }^{\beta} s_{i}(t),(i=1,2,3)
\end{gathered}
$$

自适应律设计如下:

$$
\dot{\hat{\varepsilon}}_{i}=\rho_{i} s_{i}\left|s_{i}\right|^{\alpha} \operatorname{sat}\left(s_{i}\right)
$$

稳定性证明如下: 如 (13) 式所示已经证明了李 雅普诺夫函数 $V_{1}$ 的稳定性, 现取李雅普诺夫函数 如下:

$$
V_{2}=V_{1}+\sum_{i=1}^{3} \frac{1}{2 \rho_{i}} \widetilde{\mathcal{\varepsilon}}_{i} \widetilde{\varepsilon}_{i}>0
$$

由于 $\overleftrightarrow{\varepsilon}_{i}$ 为定值, 所以不难得到, $\dot{\tilde{\varepsilon}}_{i}=\dot{\hat{\varepsilon}}_{i}-\dot{\boldsymbol{\varepsilon}}_{i}=$

$$
\begin{aligned}
\dot{V}_{2}= & \dot{V}_{1}+\sum_{i=1}^{3} \frac{1}{\rho_{i}} \widetilde{\varepsilon}_{i} \dot{\hat{\varepsilon}}_{i} \\
= & \sum_{i=1}^{3} s_{i}\left[-\hat{\varepsilon}_{i}\left|s_{i}\right|{ }^{\alpha} \operatorname{sat}\left(s_{i}\right)-\sigma_{i}\left|s_{i}\right|{ }^{\beta} s_{i}\right]+\frac{1}{\rho_{i}} \widetilde{\varepsilon}_{i} \dot{\hat{\varepsilon}}_{i} \\
= & \sum_{i=1}^{3} s_{i}\left[-\left(\tilde{\varepsilon}_{i}+\overleftrightarrow{\varepsilon}_{i}\right)\left|s_{i}\right|^{\alpha} \operatorname{sat}\left(s_{i}\right)-\right. \\
& \left.\sigma_{i}\left|s_{i}\right|{ }^{\beta} s_{i}\right]+\frac{1}{\rho_{i}} \widetilde{\varepsilon}_{i} \dot{\hat{\varepsilon}}_{i} \\
= & \sum_{i=1}^{3} s_{i}\left[-\overleftrightarrow{\varepsilon}_{i}\left|s_{i}\right|{ }^{\alpha} \operatorname{sat}\left(s_{i}\right)-\sigma_{i}\left|s_{i}\right|{ }^{\beta} s_{i}\right]+ \\
& \widetilde{\varepsilon}_{i}\left[\frac{1}{\rho_{i}} \dot{\hat{\varepsilon}}_{i}-s_{i}\left|s_{i}\right|{ }^{\alpha} \operatorname{sat}\left(s_{i}\right)\right] \\
& \text { 将公式 }(15) \text { 代人上式, 可得: } \\
\dot{V}_{2}= & \sum_{i=1}^{3} s_{i}\left[-\overleftrightarrow{\varepsilon}_{i}\left|s_{i}\right|{ }^{\alpha} \operatorname{sat}\left(s_{i}\right)-\sigma_{i}\left|s_{i}\right|{ }^{\beta} s_{i}\right]+ \\
& \widetilde{\varepsilon}_{i}\left[\frac{1}{\rho_{i}} \rho_{i} s_{i}\left|s_{i}\right|{ }^{\alpha} \operatorname{sat}\left(s_{i}\right)-s_{i}\left|s_{i}\right|{ }^{\alpha} \operatorname{sat}\left(s_{i}\right)\right]= \\
& \sum_{i=1}^{3} s_{i}\left[-\overleftrightarrow{\varepsilon}_{i}\left|s_{i}\right|{ }^{\alpha} \operatorname{sat}\left(s_{i}\right)-\sigma_{i}\left|s_{i}\right|{ }^{\beta} s_{i}\right]+0 \leqslant 0
\end{aligned}
$$

稳定性得证。

对公式(6)两端同时求导, 可以得到:

$$
\dot{s}(t)=k_{p} \dot{e}(t)+k_{i} \boldsymbol{e}(t)+k_{d} \ddot{\boldsymbol{e}}(t)
$$

结合 (14) 式可以得到:

$$
\begin{gathered}
k_{p j} e_{j}(t)+k_{i j} e_{j}(t)+k_{d j} \ddot{e}_{j}(t)= \\
k_{p j}\left(\dot{p}_{d j}-\dot{p}_{i j}\right)+k_{i j} e_{j}(t)+k_{d j} \ddot{e}_{j}(t)= \\
-\hat{\varepsilon}_{j}\left|s_{j}(t)\right|{ }^{\alpha} \operatorname{sgn}\left(s_{j}(t)\right)-\sigma_{j}\left|s_{j}(t)\right|{ }^{\beta} s_{j}(t) \\
j=(1,2,3)
\end{gathered}
$$

进一步可以得到控制信号:

$$
\begin{gathered}
\dot{p}_{i j}=\frac{1}{k_{p j}} \cdot\left[k_{p j} \dot{p}_{d j}+k_{i j} e_{j}(t)+k_{d j} \ddot{e}_{j}(t)+\right. \\
\hat{\varepsilon}_{j}\left|s_{j}(t)\right|{ }^{\alpha} \operatorname{sgn}\left(s_{j}(t)\right)+ \\
\left.\sigma_{j}\left|s_{j}(t)\right|{ }^{\beta} s_{j}(t)\right] j=(1,2,3)
\end{gathered}
$$

结合公式 (4) 和公式 (5), 可以得到各个轮的 转速:

$$
\left[\begin{array}{c}
\omega_{1} \\
\omega_{2} \\
\omega_{3} \\
\omega_{4}
\end{array}\right]=\frac{4}{R}\left[\begin{array}{cccc}
-1 & 1 & -1 & 1 \\
1 & 1 & 1 & 1 \\
\frac{1}{a+b} & \frac{-1}{a+b} & \frac{-1}{a+b} & \frac{1}{a+b}
\end{array}\right] \times
$$$$
\boldsymbol{\Gamma}^{-1}(\varphi) \times\left[\begin{array}{lll}
\dot{p_{1}} & \dot{p_{2}} & \dot{p}_{3}
\end{array}\right]^{\mathrm{T}}
$$
$\dot{\hat{\varepsilon}}_{i}$, 进而对 $V_{2}$ 求导可得: 


\section{3 仿真验证与分析}

\section{1 设计流程}

图 4 展示了本文所提控制器的设计流程。首先 对机器人的运动学进行建模, 此后为保证对此类非 线性耦合系统能够提供优越的控制性能, 本文设计 以滑模控制器为基础; 为了提高控制器的鲁棒性, 选 取了比例-积分-微分形式的滑模面; 为保证系统的 动态性能, 选取了适当的趋近律; 为了避免整定参 数, 同时使系统具有更好的自适应性, 设计了一种自 适应律。然后针对所设计的控制器在仿真中加人扰 动并与 PID 控制的普通滑模控制 (SMC) 的效果进 行了对比,最后通过实验验证的方式展示了本文所 设计控制器的实用性。

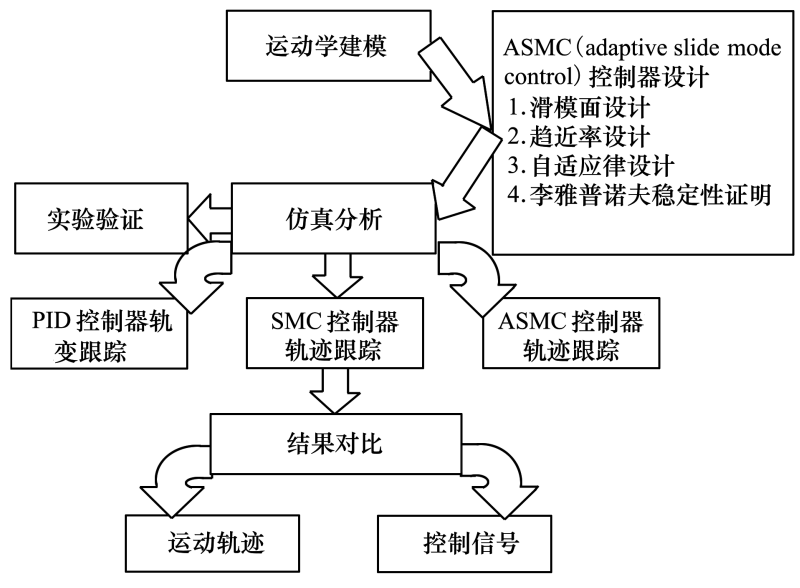

图 4 设计流程图

\section{2 仿真验证}

为验证本文所提的自适应滑动模态控制器的鲁 棒性和动态性能, 首先设计了一条轨迹, 通过对标称 轨迹的跟踪控制, 并且在其中加人了脉冲扰动, 与传 统 PID 控制器和无自适应性的 SMC 控制器进行对 比验证, 分析本文所设计控制器的性能。本文测试 使用的标称轨迹可表示如下:

$$
\left\{\begin{array}{l}
x=\frac{0.3 \cos (t / 10)}{1+\sin ^{2}(t / 10)} \\
y=\frac{0.4 \cos (t / 10)}{1+\sin ^{2}(t / 10)}, \forall t \geqslant 0 \\
\phi=0
\end{array}\right.
$$

$\left[\begin{array}{lll}x_{i} & y_{i} & \varphi_{i}\end{array}\right]^{\mathrm{T}}=\left[\begin{array}{llll}0 \mathrm{~m} & -0.2 \mathrm{~m} & 0 \mathrm{~m}\end{array}\right]^{\mathrm{T}}$ 为系 统的初始位置。同时, 为了验证系统的动态性能和
鲁棒性, 实验中加人了扰动, 在 $x$ 轴和 $y$ 轴方向分别 加人幅值为 $0.1 \mathrm{~m}$, 频率为 $0.4 \mathrm{rad} / \mathrm{s}$ 的正弦信号, 同 时在 $10 \mathrm{~s}$ 和 $30 \mathrm{~s}$ 分别加人幅值为 $0.1 \mathrm{~m}$ 和 $-0.2 \mathrm{~m}$ 的阶跃信号。仿真测试的系数设置如下:

对于 SMC 控制器和 ASMC 控制器:

$$
\left\{\begin{array}{l}
\boldsymbol{k}_{p}=\operatorname{diag}(12,12,8) \\
\boldsymbol{k}_{i}=\operatorname{diag}(4,4,1) \\
\boldsymbol{k}_{d}=\operatorname{diag}(0.02,0.02,0.02) \\
\alpha=0.8, \beta=0.065 \\
\sigma_{1}=\sigma_{2}=\sigma_{3}=0.1
\end{array}\right.
$$

在 SMC 控制器中 $\varepsilon_{1}=\varepsilon_{2}=\varepsilon_{3}=2$ 。在 ASMC 控 制器中 $\rho_{1}=1.5, \rho_{2}=4.5, \rho_{3}=1.5, \hat{\varepsilon}$ 的初值为: $\hat{\varepsilon}_{1}(0)$ $=\hat{\varepsilon}_{2}(0)=\hat{\varepsilon}_{3}(0)=0$ 。 PID 控制器的参数为:

$$
\left\{\begin{array}{l}
\boldsymbol{k}_{p}=\operatorname{diag}(12,12,8) \\
\boldsymbol{k}_{i}=\operatorname{diag}(3,2,1) \\
\boldsymbol{k}_{d}=\operatorname{diag}(1.4,1.4,1.4)
\end{array}\right.
$$

仿真结果如图 5 所示, 可以看出 3 种控制器均 能有效地抑制干扰, 由于 ASMC 初始的系数 $\varepsilon$ 为零, 因此开始时的控制效果不如其他 2 种控制器, 但随 着运动的展开,自适应律开始起到作用,在第二个阶 跃信号扰动时, ASMC 控制器的响应速度远高于其 他两种控制器, 结果见图 6 。从发生第二次脉冲扰 动到 $x$ 轴和 $y$ 轴方向偏差均小于 $0.01 \mathrm{~m}$, 所经过的 时间见表格 $1, \mathrm{ASMC}$ 控制器消耗的时间仅为 SMC 控制器的 $42.90 \%$, 仅为 PID 控制器的 $31.44 \%$ 。

表 1 控制消耗时间

\begin{tabular}{cccc}
\hline 控制器 & ASMC & SMC & PID \\
\hline 时间 $/ \mathrm{s}$ & 0.305 & 0.711 & 0.970 \\
\hline
\end{tabular}

为了更加直观地分析 3 种控制器的控制效果, 对 $x$ 轴和 $y$ 轴方向的偏差进行统计分析, 如图 7 和 图 8 所示。

通过图 7 和图 8 可以明显地看出, ASMC 控制 器虽然在控制的开始阶段, 系统的误差要大于其他 两种控制器, 但是随着时间的推移, ASMC 控制器的 控制效果逐渐改良, 并取得了优于其他 2 种控制器 的控制效果。滑模控制器的最大问题在于控制信号 的抖振, 前文中已经提到, 多幂次趋近律的引人可以 明显的改善系统抖振, 为验证其效果, 参见图 9 和 图 10。 


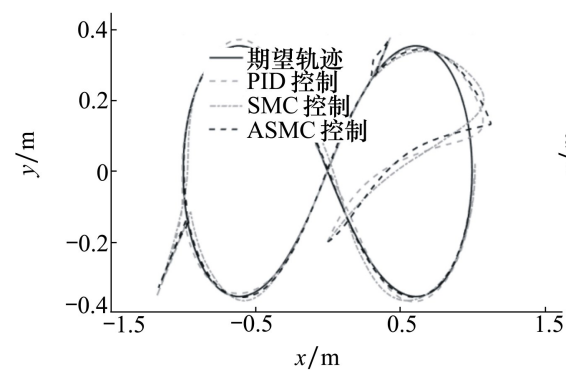

图 5 轨迹跟踪

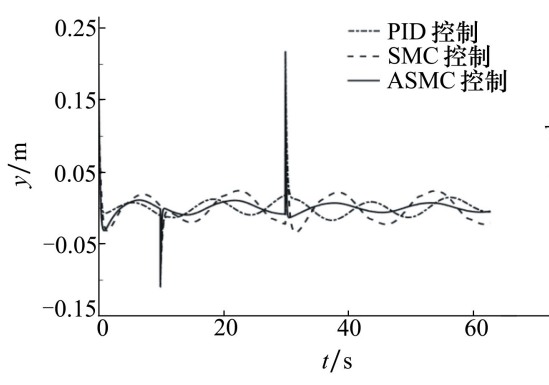

图 $8 y$ 轴偏差

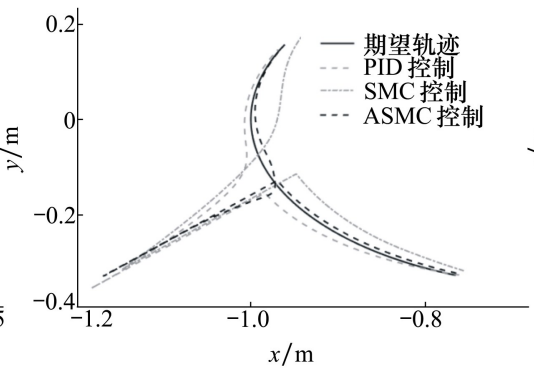

图 6 局部放大

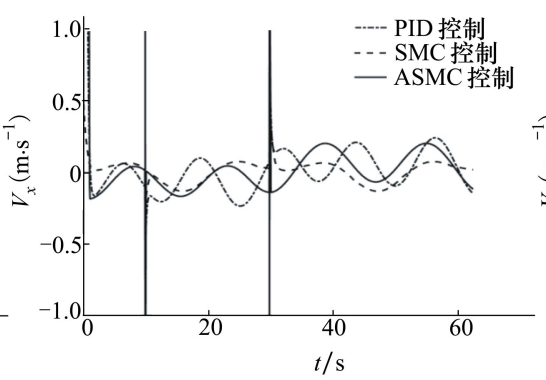

图 $9 x$ 轴速度

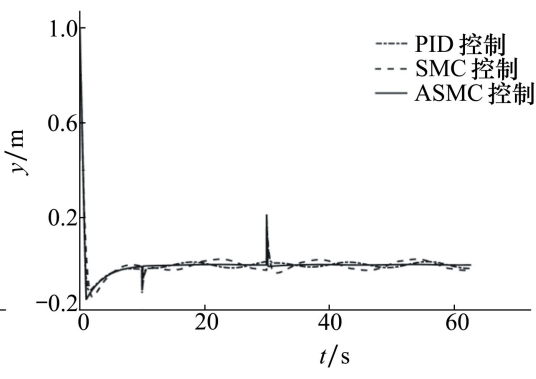

图 $7 x$ 轴偏差

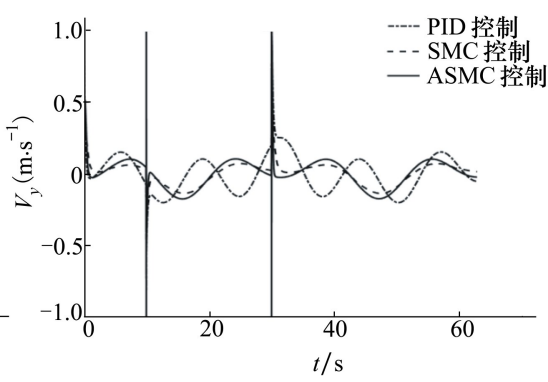

图 $10 y$ 轴速度
为准确分析各控制器性能, 本文取 $x$ 轴坐标误 差和 $y$ 轴坐标误差的均值作为控制器的控制效果, 取 $x$ 轴速度和 $y$ 轴速度的输出反映控制器的抖振情 况统计结果见表格 2 和表格 3 。

表 2 偏差平均值

\begin{tabular}{cccc}
\hline 偏差 & PID & SMC & ASMC \\
\hline$x / \mathrm{m}$ & $2.2338 \times 10^{-4}$ & $-1.9622 \times 10^{-4}$ & $-3.6746 \times 10^{-6}$ \\
$y / \mathrm{m}$ & $1.9343 \times 10^{-4}$ & $-2.0240 \times 10^{-4}$ & $-6.1646 \times 10^{-5}$ \\
\hline
\end{tabular}

表 3 控制器输出方差

\begin{tabular}{cccc}
\hline 方差 & PID & SMC & ASMC \\
\hline$x / \mathrm{m}$ & 0.261 & 0.0054 & 0.0272 \\
$y / \mathrm{m}$ & 0.0128 & 0.0054 & 0.0073 \\
\hline
\end{tabular}

对表 1 进行分析, ASMC 控制器的 $x$ 轴偏差的 平均值仅为 PID 控制器的 $1.64 \%$, 为 SMC 控制器的 $1.87 \%, \mathrm{ASMC}$ 的 $y$ 轴控制偏差为 PID 的 $31.87 \%$, 为 SMC 控制器的 $30.46 \%$, 证明本文设计的 ASMC 具有 更好的控制效果。分析表 $2, \mathrm{ASMC}$ 控制器的 $x$ 轴输 出方差为 PID 控制器的 $10.42 \%, \mathrm{ASMC}$ 的 $y$ 轴输出 方差为 PID 的 $57.03 \%$, 证明本文 ASMC 具有更好的 控制效果。此外, 本文设计的 ASMC 控制器的抖振 情况优于 PID 控制器。

\section{4 实验验证与分析}

本文的实验环境如图 11 所示, 实验样机 (轮式 机器人) 和实验所用软件如图 12 所示。

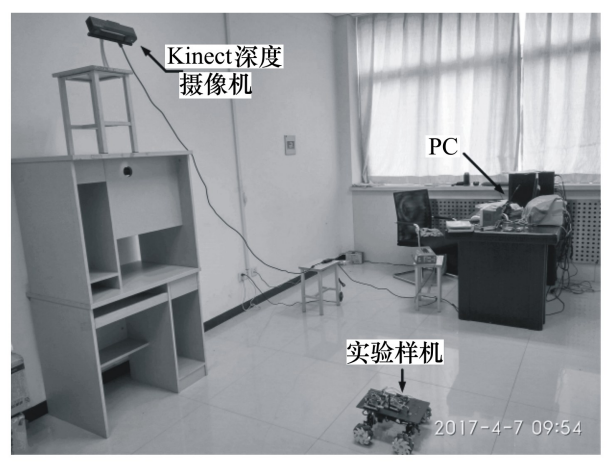

图 11 实验环境

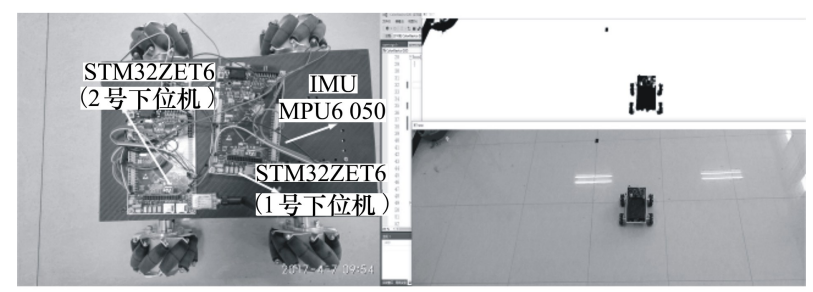

图 12 实验样机与软件 
本文实验验证设备电控流程图如图 13, 由 Kinect 深度相机连接 PC 端, 通过 USB 通信经过机 器视觉处理获得机器人的 $x$ 轴和 $y$ 轴的坐标信息, 进而通过串口将信息传输给 STM32VET6 单片机, VET6 单片机利用 SPI 通信借助 NRF24L01 通过2.4 $\mathrm{GHz}$ 通信, 将 $x$ 轴和 $y$ 轴的坐标信息传输到 STM32ZET6 单片机, 本文定义其为一号下位机, 而 与一号下位机连接的还有 IMU ( MPU6050) 用于提
供机器人的 $\phi$ 角度信息, 一号下位机获得这些信息 后, 通过与函数发生器对比产生偏差, 进而通过 ASMC 控制器产生速度控制信号, 并通过 UART 将 信号传出给另外一片 STM32ZET6, 本文定义其为二 号下位机, 二号下位机接收到速度控制信号后经过 逆运动学解算获得 4 只直流电机的期望速度, 通过 PID 控制器对电机速度进行控制, 达到控制目的。

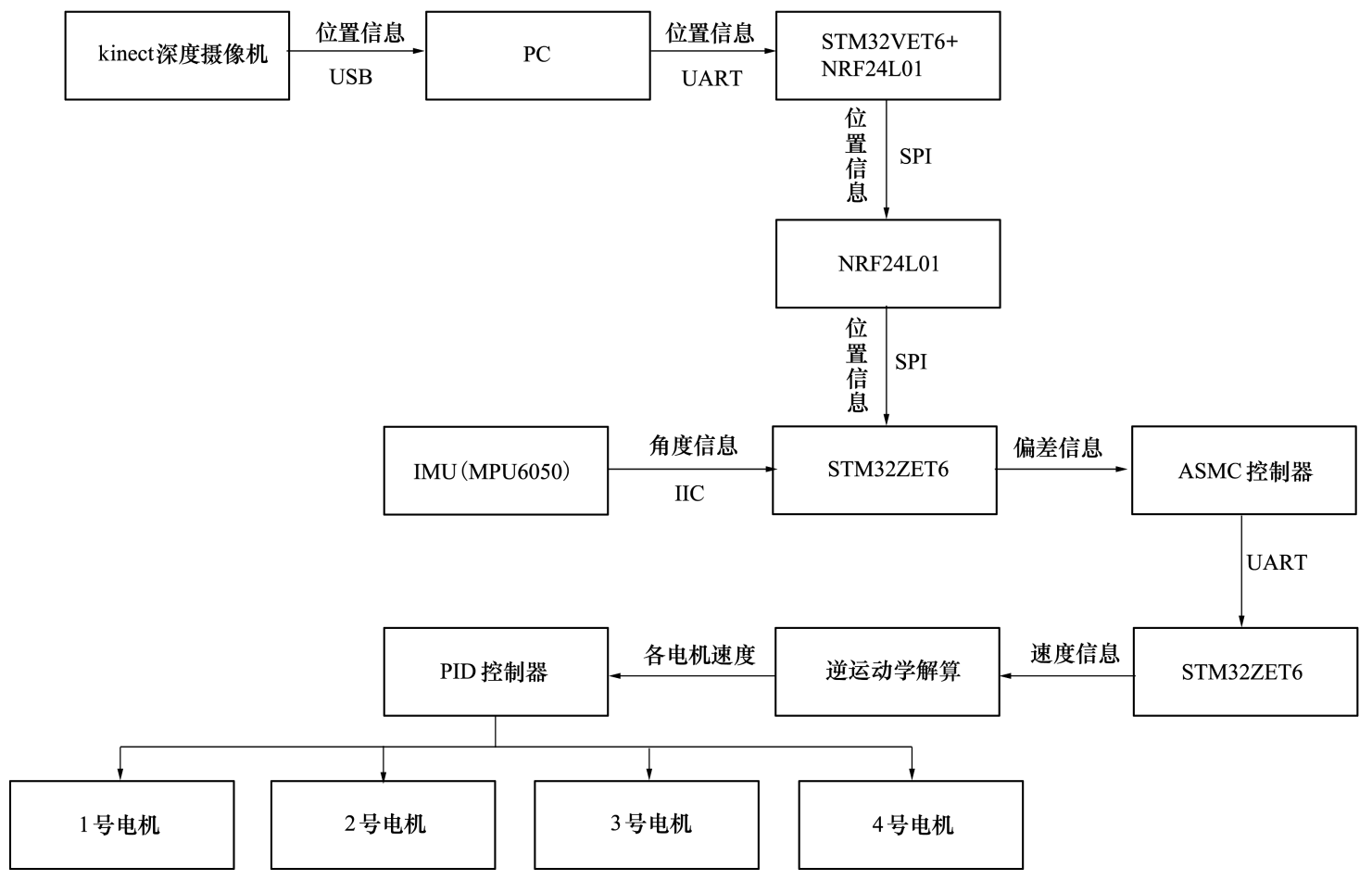

图 13 实验流程

实验验证过程中, 跟踪函数使用同 3.2 节中仿 真所用类似的标称轨迹:

$$
\left\{\begin{array}{l}
x=\frac{0.3 \cos (t / 10)}{1+\sin ^{2}(t / 10)} \times 1.5 \\
y=\frac{0.4 \cos (t / 10)}{1+\sin ^{2}(t / 10)} \times 1.5, \forall t \geqslant 0 \\
\phi=0
\end{array}\right.
$$

移动机器人初始位姿为 $\left[\begin{array}{lll}x_{i} & y_{i} & \phi_{i}\end{array}\right]^{\mathrm{T}}=$ $\left[\begin{array}{lll}0 & 0 & 0\end{array}\right]^{\mathrm{T}}$ 。通过从 kinect 相机获得的机器人位置 坐标, 绘制出轮式机器人在平面位置的跟踪曲线图

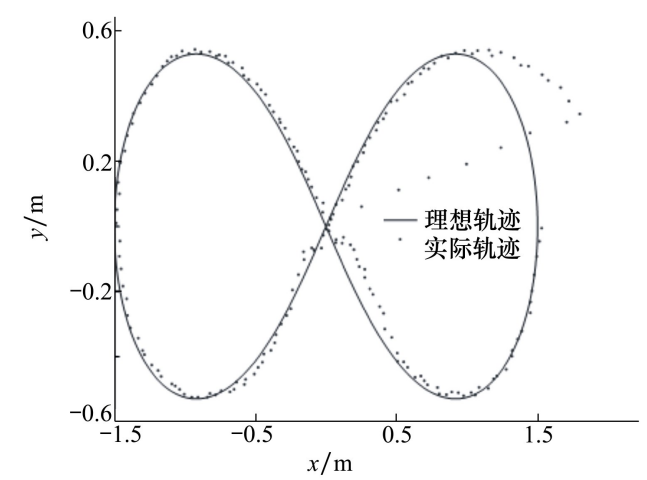

图 14 轨迹跟踪 如图 14 所示。 


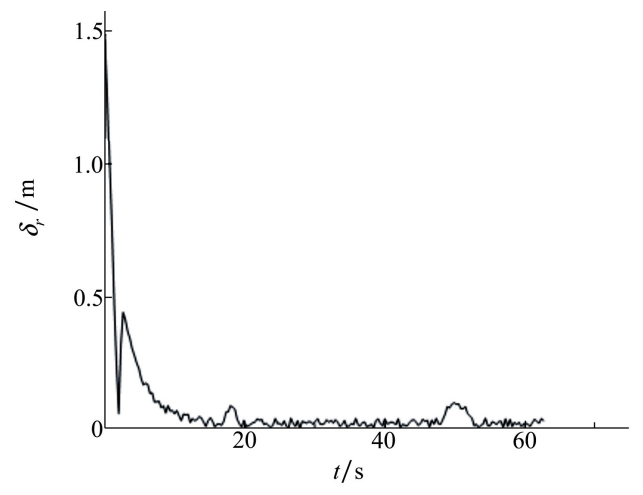

图 15 距离误差

并绘制距离误差 - 时间图像如图 15 所示, 距离 误差计算公式为 $\delta_{r}=\sqrt{\delta x^{2}+\delta y^{2}}$ 。考虑到图像处理 对机器人提取的特征具有不确定性, 并容易受外部 环境光的干扰, 因此误差图像出现小幅度波动, 整体 误差平均值为 $0.093 \mathrm{~m}$, 控制精度能够达到预想效 果。轮式机器人最终的运动轨迹图像如图 16 所示 ( 根据多帧图像合成最终图像)。

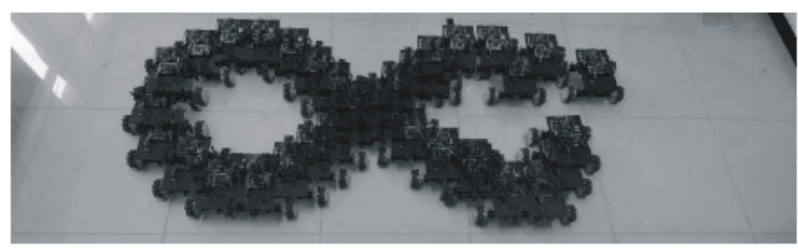

图 16 实验结果

\section{5 结 论}

本文针对麦克纳姆轮驱动的全向移动机器人的 轨迹跟踪问题, 在运动学建模的基础上设计了自适 应滑模控制器。控制器设计分为 3 个步骤: 滑模面 设计、趋近律设计以及自适应律设计。通过上述 3 个步骤完成了自适应滑模控制器的设计并利用李雅 普诺夫函数证明了其稳定性。存在脉冲扰动以及正 弦信号扰动条件下对控制器进行了仿真验证, 并将 结果与传统 PID 控制器和普通滑模控制器利用统 计学的方法进行了对比, 统计结果表明本文设计的 控制器具有响应快速, 鲁棒性强等优点。最后为验 证控制器的实用性, 构建了专用的麦克纳姆轮全向 移动机器人, 并利用 Kinect 深度摄像头和 IMU 搭建 了实验平台对机器人进行了实际的轨迹跟踪控制实 验, 结果表明该控制器能够高精度的控制机器人完 成轨迹跟踪, 证明了文中所设计控制器的可行性与 有效性。

\section{参考文献:}

[1] Martins F N, Celeste W C, Carelli R, et al. An Adaptive Dynamic Controller for Autonomous Mobile Robot Trajectory Tracking [J]. Control Engineering Practice, 2008, 16(11):1354-1363

[2] Rusu P, Petriu E M, Whalen T E, et al. Behavior-Based Neuro-Fuzzy Controller for Mobile Robot Navigation[J]. IEEE Trans on Instrumentation \& Measurement, 2002, 52(4):1335-1340

[3] Tzafestas S G. Introduction to Mobile Robot Control[ M]. London, Elsevier, 2014

[4] Muir P F, Neuman C P. Kinematic Modeling for Feedback Control of an Omnidirectional Wheeled Mobile Robot[C] // Proceedings of the IEEE International Conference on Robotics and Automation, 1987:1772-1778

[5] Tlale N, De Villiers M. Kinematics and Dynamics Modelling of a Mecanum Wheeled Mobile Platform[C] //IEEE International Conference on Mechatronics and Machine Vision in Practice, 2008:657-662

[6] Wampfler G, Salecker M, Wittenburg J. Kinematics, Dynamics, and Control of Omnidirectional Vehicles with Mecanum Wheels [J]. Mechanics Based Design of Structures and Machines, 1989, 17(2):165-177

[7] Shimada A, Yajima S, Viboonchaicheep P, et al. Mecanum-Wheel Vehicle Systems Based on Position Corrective Control[ C] // 31st Annual Conference of IEEE Industrial Electronics Society, 2005: 2077-2082

[8] Wang Z P, Yang W R, Ding G X. Sliding Mode Control for Trajectory Tracking of Nonholonomic Wheeled Mobile Robots Based on Neural Dynamic Model[C] //Second WRI Global Congress on Intelligent Systems, 2010: 270-273 
[9] Viet T D, Doan P T, Hung N, et al. Tracking Control of a Three-Wheeled Omnidirectional Mobile Manipulator System with Disturbance and Friction[J]. Journal of Mechanical Science and Technology, 2012, 26( 7):2197-2211

[10] Fierro R, Lewis F L. Control of a Nonholonomic Mobile Robot Using Neural Networks [J]. IEEE Trans on Neural Networks, $1998,9(4): 589-600$

[11] Xu D, Zhao D, Yi J, et al. Trajectory Tracking Control of Omnidirectional Wheeled Mobile Manipulators: Robust Neural Network-Based Sliding Mode Approach[J]. IEEE Trans on Systems Man \& Cybernetics Part B, 2009, 39(3) :788-799

[12] Wang J, Lu Z, Chen W, et al. An Adaptive Trajectory Tracking Control of Wheeled Mobile Robots [ C] // Proceedings of the IEEE Conference on Industrial Electronics and Applications, 2011:1156-1160

[13] Sira-Ramirez H, Barrios-Cruz E, Marquez R J. Fast Adaptive Trajectory Tracking Control of a Completely Uncertain DC Motor via Output Feedback [C] // Proceedings of the IEEE Conference on Decision and Control, 2007:4197-4202

[14] Savkin A V, Pathirana P N, Faruqi F A. The Problem of Precision Missile Guidance: LQR and $H_{\infty}$ Control Frameworks $[$ C] // Proceedings of the IEEE Conference on Decision and Control, 2001 : 1535-1540

[15] Nurdin H I, James M R, Petersen I R. Coherent Quantum LQG Control[J]. Automatica, 2009, 45( 8):1837-1846

[16] Utkin V I, Guldner J, Shi J. Sliding Mode Control in Electro-Mechanical Systems[ M]. Boca Raton CRC Press, 2009: 881-886

[17] Zhang Y, Ma G F, Guo Y N, et al. A Multi Power Reaching Law of Sliding Mode Control Design and Analysis[J]. Acta Automatica Sinica, 2013

[18] 刘金琨. 滑模变结构控制 MATLAB 仿真 $[$ M $] 2$ 版. 北京: 清华大学出版社, 2012

Liu Jinkun. Sliding Mode Control Design Matlab Simulation[M]. 2nd ed. Beijing, Tsinghua University Press, 2012 (in Chinese)

\title{
An Adaptive Robust Controller for a Mobile Robot Driven by Mecanum Wheels
}

\author{
Wang Mingming ${ }^{1,2}$, Zhu Yingying ${ }^{3}$, Zhang Lei ${ }^{1}$, Wang Lu ${ }^{1}$, \\ Wei Xuanbo ${ }^{1}$, Fang Jing ${ }^{1}$ \\ 1.School of Astronautics, Northwestern Polytechnical University, Xi'an 710072, China; \\ 2.Research Institute in Shenzhen, Northwestern Polytechnical University, Shenzhen 518057, China; \\ 3.Special Police of China, Beijing 102202, China
}

\begin{abstract}
An adaptive sliding mode controller was designed for a mobile robot driven by four mecanum wheels. The original contribution of this paper is employing adaptive robust controller to mecanum wheels driven mobile robot for obtaining better tracking and robustness performance. To complete the design of the controller, the kinematic model of the mobile robot driven by mecanum wheels was firstly proposed. An adaptive robust controller was designed subsequently. A sliding surface was designed in Proportional-Differential-Integral form, which satisfied the robustness requirements of the system. Besides, a reaching law which has quick convergence was introduced, which reduced the time consumed by the setting parameters and resisting external disturbance. The controller was demonstrated in the presence of impulsive disturbance and sinusoidal signal disturbance, which proved the superiority of the proposed controller. Finally, an experimental verification of trajectory tracking was implemented to verify the practicability and effectiveness.
\end{abstract}

Keywords : trajectory tracking; controllers, robust control, adaptive; sliding mode control; mecanum wheel 